\title{
Computer-Based Periaxial Rotation Measurement for Aligning Fractured Femur Fragments: Method and Preliminary Results
}

\author{
Ofer Ron ${ }^{1}$, Leo Joskowicz ${ }^{1}$, Ariel Simkin ${ }^{2}$, and Charles Milgrom ${ }^{2}$ \\ 1 School of Computer Science and Engineering \\ The Hebrew University of Jerusalem, Jerusalem 91904 Israel. \\ 2 Dept. of Orthopaedic Surgery, Hadassah Univ. Hospital, Jerusalem, Israel. \\ josko@cs.huji.ac.il
}

\begin{abstract}
We describe a new computer-based method for periaxial rotation measurement of healthy and fractured femurs during closed femoral fracture reduction surgery from $\mathrm{CT}$. The method provides a comparative quantitative measure to align the distal and proximal femur fragments based on periaxial rotation. We define periaxial rotation in terms of patient-specific bone features and describe an algorithm for automatically extracting these features from the CT. The algorthm extracts condyle landmarks and neck axis of the healthy bone, determines its periaxial rotation, and extrapolates this data, assuming mirror symmetry between the healthy and the fractured bone, to measure periaxial rotation between the fractured fragments. Unlike existing techniques, our method requires minimal user intervention. We applied the method to a patient data set and simulated a reduction based on the anteversion measurements with satisfactory results.
\end{abstract}

\section{Introduction}

Reducing the surgeon cumulative radiation exposure and improving the positioning accuracy are key issues in computer-assisted orthopaedic surgery. We have developed FRACAS, a computer-integrated system for closed long bone fracture reduction [5]. The system replaces uncorrelated static fluoroscopic images with a virtual reality display of $3 \mathrm{D}$ bone models created from preoperative $\mathrm{CT}$ and tracked intraoperatively in real time.

An important issue in closed medullary nailing is correctly aligning for periaxial rotation the distal and proximal fragments of the fractured femur during surgery. Precise alignment is necessary to ensure correct postoperative functionality. Currently, the surgeon performs the alignment by making a qualitative assessment of the fragments position on uncorrelated intraoperative fluoroscopic images, comparing them with preoperative X-rays of the healthy bone, and manipulating the bone fragments in an attempt to achieve a symmetric result. This procedure is lengthy, error-prone, and highly dependent on the surgeon, resulting in a non-negligible percentage of suboptimal outcomes. 


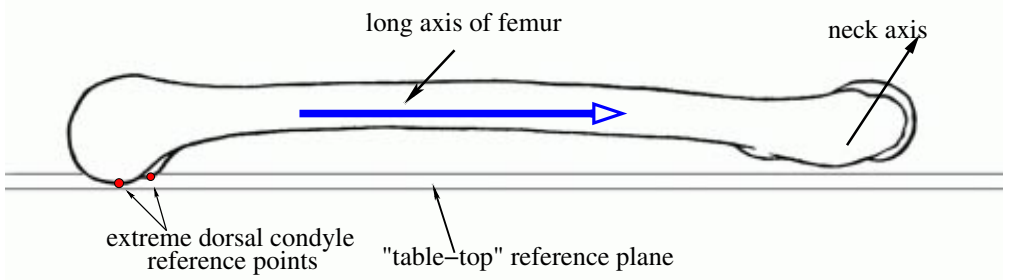

Fig. 1. Definition of periaxial rotation

We have developed a new modeling and visualization software tool for assisting surgeons in correctly aligning for periaxial rotation the distal and proximal fragments of the fractured femur during closed femoral fracture reduction surgery. The modeling module allows surgeons to preoperatively build surface models from CT data of the fractured and the healthy bone, identify and derive relevant anatomical data from these models, and define comparative periaxial rotation measurement with minimal user intervention. The visualization module presents two three-dimensional views (frontal and lateral) of the proximal and distal fragment models, a cross section of the femoral shaft of both fragments close to the fracture location, and their anatomical axes whose relative positions are updated in real time from position data obtained from an optical tracking device. The measure of their periaxial rotation is displayed and updated, providing a comparative patient-specific quantitative measurement to evaluate the quality of the alignment. The surgeon utilizes the views and the periaxial rotation value to bring the bone fractures into alignment without further use of fluoroscopy.

In this paper, we describe the computer-based method for periaxial rotation measurement of healthy and fractured femurs. We define periaxial rotation in terms of patient-specific bone features and describe an algorithm for automatically extracting these features from the CT. Periaxial rotation is the angle between the axis of the femoral neck and the "table top" reference plane, which is the plane passing through the extreme dorsal points of the medial and lateral condyles and parallel to the main axis of the femur (Figure 1). By spatially relating the proximal and distal fragments, this definition allows the extension of periaxial rotation measurements to the fractured femur, which could not be obtained with existing methods. The algorithm extracts condyle landmarks and neck axis of the healthy bone, determines its periaxial rotation, and extrapolates this data, assuming mirror symmetry between the healthy and the fractured bone, to measure periaxial rotation between the fractured distal and proximal fragments. In contrast with existing techniques, our method requires minimal user intervention in the modeling and measurement process. 


\section{Previous Work}

Periaxial rotation measurement is closely related to anteversion measurement. Most previous research addresses the problem of establishing methods for accurately measuring anteversion using X-rays, CT scans, and computer reconstructed models. The main difficulty is that the measure of femoral anteversion is not uniquely defined, and there are discrepancies as to what is best way to measure femoral anteversion. Consequently, most of the efforts have been focused on creating new measurement protocols using CT scans or X-Rays.

Hoftstatter et al 4 describe a system to assist surgeons in femoral fracture reduction and periaxial rotation correction based on fluoroscopic images. Periaxial rotation is defined from manually selected landmarks in AP and lateral fluoroscopic images and is updated in real time during the procedure. The long axis of the healthy femur and fractured femur are identified independently, so they do not account for the arching of the femur. The drawbacks of this method are the manual landmark selection and the inaccurate fragment axes definition.

Egund and Palmer [1] describe a method which consists of acquiring several CT slices at selected locations, manually extracting geometric features from those slices, defining a reference plane and a plane though the femur head from the features, and measuring anteversion as the angle between the plane normals. Herman and Egund [3] propose to measure anteversion from three CT slices and from fluoroscopic images of the whole femur at precise predetermined viewpoints. The method requires significant manual user intervention. Herman and Egund [2] determine that the femur positioning on CT slices does influence the femoral anteversion measurement, and conclude that a full 3D reconstruction of the bone from the CT scans is necessary to compensate for non standard bone positioning.

Comparison studies of alternative methods of measuring femoral anteversion with radiographic methods have also been conducted. Murphy [7] compares four different definitions of anteversion measurement and conclude that the "table top" method for locating the condylar plane (which we refer to as the table top plane), is the simplest, most reproducible, and is most similar to the clinical method of measurement. Sugano [8] compares various definitions and measurement procedures based on different data for anteversion. They provide a precise statistical evaluation of each of the methods by comparing their results to an anteversion value (termed "true anteversion") measured by positioning a 3D model reconstructed from $\mathrm{CT}$ slices of the femur in a position close to the the classical clinical method of measuring anteversion manually. They conclude that most methods either overestimate or underestimate the average and standard deviation of the method utilizing the full $3 \mathrm{D}$ reconstruction.

In contrast with previous approaches, our work aims at providing a comparative measure of femur anteversion before and after the fracture, rather than attempting to find an absolute measure of anteversion or periaxial rotation only applicable to healthy femurs. We believe that this relative measure is the most useful one for restoring function and improving surgical outcomes. The emphasis is on precise and robust automatic location of geometric features in the bone models. 


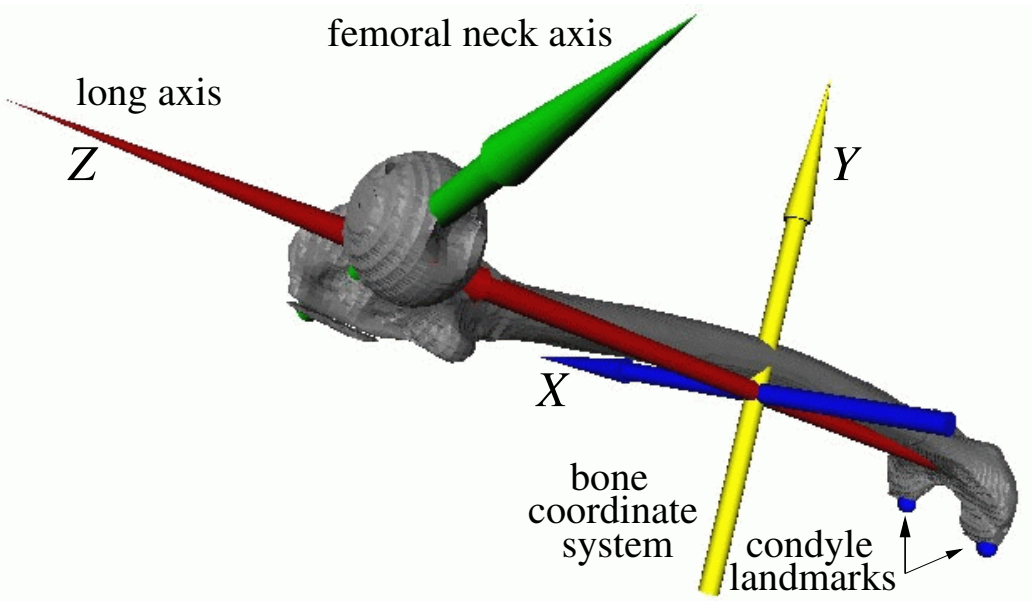

Fig. 2. Healthy femur feature extraction

\section{Our Method}

The inputs are three surface bone models (triangular surface meshes) of the healthy femur and the distal and proximal fragments of the fractured femur. The models can be readily obtained from CT data using standard segmentation and surface construction techniques, [5]. The output is a set of relevant geometric features (femur main axes, condyle landmarks, femur head and neck axis) and a measure of periaxial rotation.

The method consists of three steps. First, we extract the geometric features of the healthy femur and compute from them its periaxial rotation (Figure 2). Next, we extract the geometric features of the fractured femur proximal and distal fragments (Figure 3) by using the healthy femur features as reference (Figure 4). Finally, we use this information for accurate alignment of proximal and distal fragments during surgery. We briefly describe each step next.

The periaxial rotation value for the healthy bone is obtained in two steps (Figures 2 and 3). First, we compute the principal axes of the healthy femur, and the proximal and distal fragments of the fractured femur using the Principal Axis Transformation (PAX) technique [9]. PAX computes the three major orthogonal axes of mass distribution of a solid object. The principal axis is the one with the highest mass distribution (largest eigenvalue). We then manually orient the axes according to a preferred orientation, which can be chosen arbitrarily. This establishes a reference frame for the location of the model vertices and geometric features. Correct orientation of the principal axes is crucial to the correctness of the computations, and thus has to be set by the user. Orientations are defined for bones whose condyles are touching the table (healthy bone and distal fragment), or whose lesser trochanter is touching the table (proximal fragment). The long axis $(Z)$ is parallel to the table top and oriented toward the femur head, away 


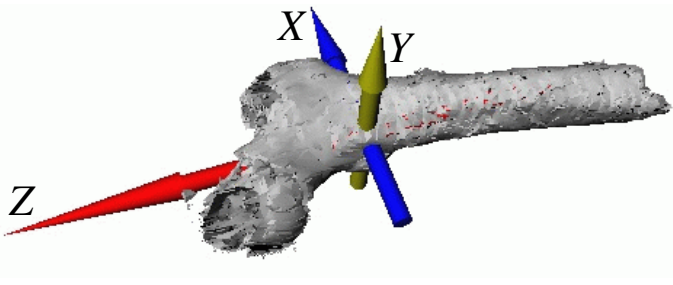

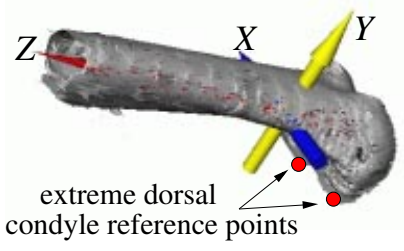

Fig. 3. Features of proximal (left) and distal (right) fragments

from the condyles. The second axis parallel to the table top $(X)$ is always oriented in the opposite direction from the femural head. The axis orthogonal to the table top $(Y)$ is always oriented upwards. This axes orientation defines a right (left) axis system for the right (left) leg bones, and embodies our assumption that the left and right femurs of a normal patient are approximately mirror-symmetric about the $Y Z$ plane. We use this new coordinate system with its origin at the centroid of the model for all subsequent computations.

Second, we extract the relevant geometric features from the healthy bone: the two extreme dorsal condyle points and the axis passing through the femur's neck. The extreme dorsal condyle points are the lowest points with respect to the $Y$ axis which lie on opposite sides of the $Y Z$ plane. The femoral neck axis is the principal axis found by PAX on the region upwards of the lesser trochanter (femur head and neck). Model vertices in this region are found by "cutting" the femur twice above the fracture twice with a plane parallel to the $X Y$ plane. Finally, we compute the periaxial rotation from the condyle landmarks and the femur neck axis as the angle between the plane normal and the femur neck axis.

The relevant geometric features of the fractured femur fragments are extracted in an identical manner. The condyle landmarks and long axis are extracted for the distal fragment, and the femoral neck axis for the proximal. We approximate the location of the fracture on the healthy bone by assuming mirror symmetry between healthy and fractured bone, splitting the healthy bone model at that location, and calculating the principal axes for the interpolated distal fragment (Figure 4). We calculate the rigid transformation that takes the interpolated distal fragment coordinate system to the real distal fragment coordinate system. We then transform the femoral long axis of the healthy femur to the distal fragment coordinate system (as given by PAX) using this transformation, defining it as the interpolated long axis of the fractured femur. We then define the "table top" reference plane for the distal fragment as the plane containing the distal fragment condyle landmarks and parallel to the interpolated long axis.

The periaxial rotation value is computed and updated in real time during surgery by using the known location of the proximal and distal fragments in world coordinates to relate the table top plane defined for the distal fragment to the neck axis of the proximal fragment. The periaxial rotation value is computed for any spatial configuration of the fragments as their relative positions change. 


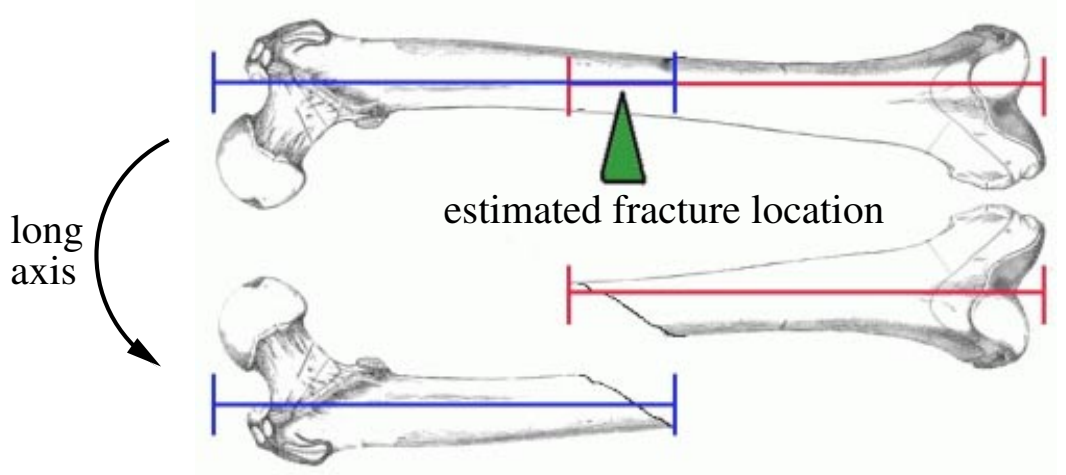

Fig. 4. Fracture extrapolation on healthy bone

The advantages of our method are several. First, no additional images are required. The bone models are the same models used for navigation during surgery, which are derived from the preoperative CT scan. Second, no manual CT slice selection is required. The method automatically identifies the slices belonging to regions of interest where geometric features will be extracted. Third, the feature extraction on both the healthy and the fractured femur is fully automatic, which ensures robustness, reproducibility, and accuracy. Fourth, the method is position scan independent. The computation of the periaxial rotation is independent of the scanning position of both femurs, since feature extraction is relative and coordinate transformations for matching them are computed explicitly.

\section{Experimental Results}

The main difficulty in designing experiments to validate our approach is that there is no "golden standard" for determining the correct absolute value of the periaxial rotation. Instead, we designed experiments to determine the usefulness of the method in fracture reduction. We have obtained scans of several actual fracture cases and dry femurs and have performed periaxial rotation measurements on them. When only one dry femur is available, we create a mirror image and "break" it to obtain distal and proximal fragments.

To determine the effectiveness of the method, we have performed the following in-vitro experiment using a whole dry femur and a physical fracture simulator device [6]. First, we CT scan the femur and construct a surface model of it. Then, we break the femur into two fragments, CT scan both fragments, and construct reflected models of them. We extract the geometric features and compute periaxial rotation on the three models as described above. We then a attach an optical tracking instrument to each fragment, register each to its model using contactbased registration techniques [10], and cover both fragments with a cloth, so that they can be manipulated but not seen. We perform fragment alignment using 
the visualization software and the periaxial rotation information only. Once the alignment has been performed according to the software, we compare it with the actual physical position of the bone fragments and ask a surgeon to determine if the discrepancy is acceptable. While the comparison is only qualitative, the initial results are encouraging.

We are planning on carrying the first in-vivo test in the near future. We will use the traditional fluoroscopic validation method to qualitatively evaluate if the periaxial rotation measurement combined with the visualization method reduces the reduction time and provides satisfactory results.

\section{References}

1. Egund N., Palmer J., 1984, "Femoral Anatomy Described in Cylindrical Coordinates Using Computed Tomography". Acta Radiologica Diagnosis, vol. 25, pg. 209-215.

2. Hermann K.L., Egund N., 1997, "CT Measurement of Anteversion in the Femoral Neck: The Influence of Femur Positioning". Acta Radiologica, vol 38,pg. 527-532.

3. Hermann K.L., Egund N., 1998, "Measuring Anteversion in the Femoral Neck from Routine Radiographs". Acta Radiologica, vol 39,pg. 410-415.

4. Hofstatter, R., Slomczykowski, M., Krettek, C., et al. 2000, "Computer-Assisted Fluoroscopy-based reduction of femoral fractures and antetorsion correction", $J$. of Computer-Aided Surgery, Vol 5(5).

5. Joskowicz, L., Milgrom, C., Simkin, A., Tockus, L., Yaniv, Z., 1999, "FRACAS: A System for Computer-Aided Image-Guided Long Bone Fracture", Journal of Computer-Aided Surgery, Vol 3(6), May.

6. Joskowicz, L., Milgrom, C., and Simkin, A., 2000, "Simulator and Distal Targeting Device for In-Vitro Experimentation and Training in Computer-Aided Closed Medullary Nailing", Proc. 14th Int. Congress on Computer-Assisted Radiology and Surgery, CARS'2000, Lemke et. al. eds, Elsevier, pp 969-975.

7. Murphy, S., Simon S., Kijewski, P., et al., 1987, "Femoral Anteversion". The Journal of Bone and Joint Surgery, Inc., vol 69-A, pg. 1169-1176.

8. Sugano N., , Noble, P., and Kamaric, E., 1998, "A Comparison of Alternative Methods of Measuring Femoral Anteversion".Journal of Computed Assisted Tomography, vol 22(4), pg. 610-614.

9. Tsao J., Chiodo, C., Williamson, D., Wilson, M., and Kikinis, R., 1998, "ComputerAssisted Quantification of Periaxial Bone Rotation from X-Ray CT".Journal of Computed Assisted Tomography, vol. 22(4), pg. 615-620.

10. Yaniv, Z., Sadowsky, O., and Joskowicz, L., 2000, "In-Vitro Accuracy Study of Contact and Image-Based Registration: Materials, Methods, and Experimental Results", Proc. 14th Int. Congress on Computer-Assisted Radiology and Surgery, CARS'2000, Lemke et. al. eds, Elsevier, pp. 141-146.

\section{Acknowledgement}

This research was supported in part by a grant from the Israel Ministry of Industry and Trade for the IZMEL Consortium on Image-Guided Therapy. We thank Ofri Sadowski and Ziv Yaniv for their help in formulating the ideas presented in this paper. 